chenmolekül CD19 werde bei B-ZellNeoplasien wie dem DLBCL stark exprimiert und begünstige Überleben und Proliferation der Lymphomzellen, erläuterte Kami J. Maddocks, Columbus, $\mathrm{OH} /$ USA. MOR208 ist ein humanisierter monoklonaler Antikörper gegen CD19, dessen antitumorale Effekte über eine Antikörper-abhängige, zellvermittelte Zytotoxizität und Phagozytose zustande kommen. Darüber hinaus wirkt MOR208 direkt zytotoxisch. Kombiniert mit Lenalidomid wird eine synergistische antitumorale Wirkung erreicht.
Das Regime aus MOR208 plus Lenalidomid wurde in der Phase-II-Studie LMIND bei bislang 44 älteren Patienten mit rezidiviertem oder refraktärem DLBCL geprüft. Die Teilnehmer waren für eine Hochdosis-Chemotherapie und Stammzelltransplantation nicht geeignet und intensiv vorbehandelt (1-3 Vortherapien einschließlich Rituximab). Die Kombination wurde ein Jahr lang verabreicht; Patienten mit kontrollierter Erkrankung erhielten den Antikörper danach als Monotherapie weiter. Auf die Kombination sprachen $56 \%$ der Patien-

\section{Verschiedene Wege zum therapiefreien Leben mit CML}

Eine Reihe von Absetzstudien belegt, dass ein Teil der Patienten mit chronischer myeloischer Leukämie (CML) hoffen kann, bei einem stabilen tiefen Ansprechen auf die Therapie mit einem Tyrosinkinaseinhibitor (TKI) zumindest einige Jahre ohne Therapie leben zu können.

Den 96-Wochen-Daten der ENESTfreedom-Studie zufolge waren zu diesem Zeitpunkt 93 der 190 Patienten (48,9\%) nach Absetzen einer Erstlinientherapie mit Nilotinib weiter in stabiler therapiefreier Remission (TFR) [Ross D et al. EHA. 2017;Abstr P601]. Bei 97 von insgesamt 190 Patienten, die den TKI abgesetzt hatten, kam es zu einem Verlust der MMR ("major molecular response"). 9 Patienten beendeten die Studie vorzeitig, von den übrigen 88 erreichten unter einer erneuten Behandlung mit Nilotinib 98,9\% wieder eine MMR, $92 \%$ auch wieder eine $M^{4,5}$ (BCR-ABL/ABL $\leq 0,0032 \%$ ). Die 96-Wochen-Daten der ENESTop-Studie, in der das Absetzen von Nilotinib nach vorangegangener Imatinib-Behandlung untersucht wird, bestätigten diese Ergebnisse auch in der Zweitlinie. Von 126 Patienten, die die Absatzkriterien erfüllten, wiesen 67 (53,2\%) immer noch eine TFR auf. 52 von 56 Patienten, die nach dem Verlust der MMR erneut Nilotinib erhalten hatten, erreichten wieder mindestens eine $\mathrm{MR}^{4}$ (BCR-ABL/ABL $\left.\leq 0,01 \%\right)$. In beiden Studien hatten muskuloskelettale Symptome nach Absetzen zugenommen, klangen aber in den zweiten 48 Wochen der Nachbeobachtung wieder ab.

In der britischen Destiny-Studie wird bei Patienten mit mindestens dreijähriger TKI-Therapie und mindestens einer MMR eine schrittweise Therapiereduktion ge- testet. Der jeweilige TKI wird für $12 \mathrm{Mo-}$ nate in der Dosis halbiert (Imatinib 200 $\mathrm{mg} /$ Tag, Dasatinib 50 mg/Tag oder Nilotinib 200 mg 2-mal täglich) und erst dann - bei weiter erhaltenem tiefem Ansprechen - gestoppt. Die 2-Jahres-Daten zeigen in der Gruppe mit zuvor stabiler $M R^{4}$ ein rezidivfreies Überleben (RFS) von im Vergleich zu anderen Studien sehr guten $77 \%$, was nach Meinung der Forscher für dieses stufenweise Vorgehen sprechen könnte [Clark R et al. EHA. 2017;Abstr S423]. Bei Patienten mit MMR, aber ohne MR 4 lag die RFS-Rate bei $39 \%$, sodass eventuell auch diesen Patienten ein zumindest zeitweises Absetzen ermöglicht werden könnte. Ein wichtiger prognostischer Faktor für den Erhalt des tiefen Ansprechens war die Dauer der vorangegangenen Therapie. Ein Progress trat in keinem Fall auf, nur ein Patient verlor sein hämatologisches Ansprechen. Italienische Hämatoonkologen befragten 120 Patienten mit langjährig behandelter CML nach ihrer Meinung zum Absetzen [Riva S et al. EHA. 2017;Abstr P729]. Etwa 4 von 5 Patienten fanden ein Absetzen eine interessante Option, wenn eine lang anhaltende Remission zu erwarten ist. Die am häufigsten geäußerten Bedenken waren ein mögliches Rezidiv (60,5\%), die Angst vor einer Resistenz bei erneuter TKI-Einnahme $(44,5 \%)$ und die Angst, die Familie oder Freunde zu enttäuschen (26,9\%). Ältere Patienten hatten mehr Angst vor einem Rezidiv und nachfolgend mangelndem Therapieansprechen als jüngere.

Friederike Klein

Bericht vom 22. Kongress der European Hematology Association vom 22. bis 25. Juni 2017 in Madrid, Spanien ten an: $32 \%$ erreichten eine komplette, $24 \%$ eine partielle Remission und weitere $12 \%$ eine Krankheitsstabilisierung. Remissionen traten schnell nach median 1,6 Monaten ein und hielten meist langfristig an. Zudem wurde der Antikörper gut und ohne Auftreten unerwarteter Nebenwirkungen oder Infusionsreaktionen vertragen. Zwecks besserer Tolerabilität wurde allerdings bei etwa einem Viertel der Patienten die LenalidomidDosis reduziert. Die vorläufigen Ergebnisse sind ermutigend. Deshalb sollen jetzt weitere Patienten für die Phase-IIStudie rekrutiert werden.

\section{Infektionen bleiben häufigste Komplikation beim Myelom}

Patienten mit MM sind auch in der Ära neuer Substanzen weiterhin stark infektionsgefährdet. Das zeigt eine retrospektive Analyse von 479 MM-Patienten, die zwischen 2003 und 2014 am Universitätsklinikum Jena behandelt wurden [von Lilienthal-Toal M et al. EHA. 2017; Abstr P647]. Die median 62 Jahre alten Patienten hatten mehrheitlich eine fortgeschrittene Erkrankung und erhielten sowohl konventionelle Langzeit-Chemotherapien als auch Induktions-Chemotherapien, Chemotherapien zur Stammzell-Mobilisation und Hochdosis-Melphalan-Therapien inklusive Stammzelltransplantation.

Insgesamt traten im Auswertungszeitraum 773 Infektionen auf. $65 \%$ der Patienten erlitten zumindest eine infektiöse Episode. Nur 35\% erkrankten nicht an einer Infektion, darunter auch 25 Patienten mit Melphalan-Hochdosistherapie. In $72 \%$ der Fälle handelte es sich um bakterielle Infektionen; außerdem wurden in 37 Fällen Herpes-Zoster-Infektionen registriert. Mykosen waren extrem selten.

Wichtigste Risikofaktoren für eine Infektion waren hohe Tumorlast (Odds Ratio [OR] 1,5), Rezidivsituation (OR 2,3) und die Hochdosis-Melphalan-Therapie (OR 11,2). Die Infektionsrate blieb im Auswertungszeitraum relativ stabil. Es sei bedauerlich, so die Forscher, dass die Inzidenz dieser Komplikationen trotz der insgesamt verbesserten Behandlung des MM bislang nicht gesenkt werden konnte. Katharina Arnheim

Bericht vom 22. Kongress der European Hematology Association vom 22. bis 25. Juni 2017 in Madrid, Spanien 